\title{
RELAÇÕES ENTRE PROPRIEDADES FÍSICAS E CARACTERÍSTICAS DE SOLOS DA REGIÃO SUL DO BRASIL ${ }^{(1)}$
}

\author{
N. F. B. GIAROLA(2), A. P. SILVA ${ }^{(3)} \&$ S. IMHOFF(4)
}

\begin{abstract}
RESUMO
Relações funcionais entre atributos dos solos podem ser utilizadas para associar certas propriedades físicas com características desses solos. Este trabal ho teve como objetivo determi nar as relações entre algumas propriedades físi cas dos solos, especificamente densidade do solo (Ds), densi dade de partículas $(D p)$ e capacidade de campo $\left(\theta_{\mathrm{Cc}}\right)$, ponto de murcha permanente $\left(\theta_{\mathrm{PMP}}\right)$ e água disponível $\left(\theta_{A D}\right)$ e atributos rotineiramente determinados em levantamentos pedológicos. No estudo, foram utilizados 18 perfis representativos dos solos ocorrentes nos estados do Rio Grande do Sul e Santa Catarina, região Sul do Brasil. Foram coletadas amostras deformadas e indeformadas nos horizontes $A$ e B para a determinação de Ds, $D p, \theta_{C C}, \theta_{P M P}$ e $\theta_{A D}$, granulometria, matéria orgânica, óxidos de ferro e alumínio. As relações obtidas (regressões múltiplas) demonstraram que $D s, D p, \theta_{C C}, \theta_{\mathrm{PMP}}$ e $\theta_{\mathrm{AD}}$ foram influenciadas por um número reduzido de características dos solos, especificamente teores de argila, silte e teor de óxidos de ferro e alumínio. Esses resultados preliminares podem servir para nortear o desenvolvimento de funções de pedotransferência para a região Sul do Brasil.
\end{abstract}

Termos de indexação: atributos físi cos do solo, atributos químicos do solo, análise de regressão múltipla.

(1) Recebido para publicação em janeiro de 2002 e aprovado em agosto de 2002.

(2) Professora do Departamento de Ciências Agrárias, U niversidade Estadual do Oeste do Paraná - UNIOESTE. Rua Pernambuco 1777, CEP 85960-000 Marechal Rondon (PR) e Doutoranda em Solos e Nutrição de Plantas, Departamento de Solos e Nutrição de Plantas. Escola Superior de Agricultura “Luiz de Queiroz" - ESALQ/USP. Av. Pádua Dias 11, CEP 13400-970 Piracicaba (SP). E-mail: neydef@unioeste.br

(3) Professor do Departamento de Solos e Nutrição de Plantas da Escola Superior de Agricultura "Luiz de Queiroz" - ESALQ/USP. E-mail: apisilva@esalq.usp.br

(4) Professora da Universidad Nacional del Litoral (Argentina) e Doutoranda em Solos e Nutrição de PIantas, ESALQ/USP. E-mail: simhoff@fca.unl.edu.ar 


\title{
SUMMARY: RELATIONSHIPS BETWEEN PHYSICAL SOIL PROPERTIES AND CHARACTERISTICS OF SOUTH BRAZILIAN SOIL
}

\begin{abstract}
Functional relations between soil attributes may beused to associatedifferent physical soil properties with soil characteristics. The objective of this work was to determine the retationships between somesoil physical properties, namely bulk density (BD), particledensity (PD), field capacity $\left(\theta_{\mathrm{FC}}\right)$, permanent wilting point $\left(\theta_{\mathrm{PWP}}\right)$ and availabl ewater capacity $\left(\theta_{\mathrm{AD}}\right)$ with soil attributes measured in routine soil surveys. Eighteen representative soil profiles from Rio Grande do Sul and Santa Catarina State, Southern Brazil, were used in this study. Disturbed and undisturbed soil samples were collected from A and B horizons to quantify $\mathrm{BD}, \mathrm{PD}, \theta_{\mathrm{FC}}, \theta_{\mathrm{PWP}}$ and $\theta_{\mathrm{AD}}$, particle size, organic matter, iron, and aluminum oxides. Multiple regressions demonstrated that $\mathrm{BD}, \mathrm{PD}, \theta_{\mathrm{FC}}, \theta_{\mathrm{PWP}}$ and $\theta_{\mathrm{AD}}$ were related with some soil characteristics, chiefly clay, silt and iron oxide contents. These preliminary results may help direct further studies on pedotransfer functions for Southern Brazil.
\end{abstract}

Index terms: physical soil attributes, chemical soil attributes, multipleregression analysis.

\section{INTRODUÇÃO}

Relações funcionais podem ser utilizadas para estimar propriedades físicas do solo a partir de características facilmente mensuráveis. Relações quantitativas entre a capacidade de campo, ponto de murcha permanente e a densidade do sol o com a textura do solo foram determinadas por Arruda et al. (1987), para sol os de São Paulo. Outras relações entre Ds egranulometria e carbono orgânico também foram obtidas por Bernoux et al. (1998), para solos da Bacia Amazônica. A evolução dessas relações consiste no desenvolvimento de funções mais abrangentes denominadas "funções de pedotransferência".

As funções de pedotransferência (FPTs) são métodos utilizados para estimar atributos de solo a partir de dados de fácil aquisição (Bouma, 1989). As FPTs são úteis para transformar, por meio de regressões ou relações funcionais, a informação básica encontrada nos levantamentos de sol os numa informação de aplicação mais ampla, tal como na modelagem de processos no solo (Wagenet et al ., 1991; Bernoux et al., 1998).

As funções de pedotransferência foram utilizadas para estimar propriedades físicas do solo, como condutividade hidráulica eretenção deágua, a partir da distribuição do tamanho de partículas, conteúdo de matéria orgânica e densidade do solo (Tietje \& Tapkenhinrichs, 1992). Van den Berg et al. (1997) desenvolveram FTPs para modelar a retenção de água em Ferralsols/Oxisols da África, Ásia eAmérica do Sul. Nesse trabalho, os parâmetros da equação devan Genuchten (1980) foram estimados utilizando val ores da distribui ção do tamanho de partículas, Fe eAl ditionito, teor de carbono orgânico e capacidade de troca de cátions. Recentemente, no Brasil, FTPs foram desenvolvidas e validadas por Tomasella et al. (2000), utilizando dados de 500 horizontes de sol os coletados nas regiões Norte, Nordeste e Sudeste. Essas FTPs permitem predizer os parâmetros da equação de van Genuchten.

O número de amostras para desenvolver FPTs deve ser elevado, para que as estimativas sejam representativas e confiáveis (Pachepsky \& Rawls, 1999), o que, às vezes, torna o processo difícil e oneroso. Além disso, a qualidade de uma FTP pode ser mel horada, se forem definidas relações prévias com um número menor de amostras que indiquem as variáveis com maior grau de associação. A partir dessas relações, o número, o local de amostragem e as variáveis a serem analisadas podem ser mais bem definidas.

$\mathrm{Na}$ literatura, poucos trabalhos abordam as relações funcionais entre propriedades físicas e características de solos da região Sul do Brasil. Perfis de solos que abrangem a variação dos tipos de solos ocorrentes nessa região foram apresentados e discutidos naVI Reunião deCorrelação, Classificação eAplicação de Levantamentos de Sol os (2000). Esse banco de perfis pode ser usado para estabelecer relações quantitativas entre atributos de solo.

O objetivo deste trabalho foi determinar as relações entre al gumas propriedades físicas do solo, especificamente densidade do solo, densidade de partículas e retenção deágua, e os atributos de sol os representativos da região Sul do Brasil, com vistas em prover uma base de informações para orientar a tomada de decisão nos estudos de desenvol vimento de funções de pedotranferência, quanto à importância das variáveis analisadas.

\section{MATERIAL E MÉTODOS}

Neste estudo, foram utilizadas amostras deformadas e indeformadas dos horizontes A e B 
provenientes de 18 perfis de solos representativos de diversos locais dos estados de Santa Catarina e Rio Grande do Sul (Quadro 1), apresentados e discutidos na VI Reunião de Correlação, Classificação eA plicação de Levantamentos de Solos (2000), cujos resultados analíticos encontram-se no quadro 2. A classificação dos solos foi efetuada de acordo com EMBRAPA (1999).

As amostras deformadas foram secas ao ar e passadas por peneira com abertura de malha de $2 \mathrm{~mm}$. A fração $<2 \mathrm{~mm}$ foi utilizada para as seguintes análises: distribuição do tamanho de partículas, usando a mistura hexametafosfato de sódio + hidróxido de sódio 0,1 N como agente dispersante (IAC, 1986); o método da tami sação, para separar as cinco frações areia (0,053-2 mm), e o método do densímetro, para determinar silte (2-50 $\mu \mathrm{m})$ eargila $(<2 \mu \mathrm{m})$; teor de carbono orgânico, de acordo com Walkley/Black; óxidos $\mathrm{Al}_{2} \mathrm{O}_{3}$ e $\mathrm{Fe}_{2} \mathrm{O}_{3}$, determinados após digestão com $\mathrm{H}_{2} \mathrm{SO}_{4} 1: 1$, conforme Vettori (1969) e EMBRAPA (1979). A retenção de água no ponto de murcha permanente $\left(\theta_{\text {PMP }}\right)$, foi realizada aplicando-se um potencial mátrico $(\Psi)$ de -1,5 $\mathrm{MPa}$, em panelas de pressão de Richards, conforme Klute (1986), também foi determinada em amostras deformadas.

A retenção de água na capacidade de campo $\left(\theta_{c c}\right)$ foi medida em amostras com estrutura indeformada, coletadas em anéis cilíndricos de aço inox de $100 \mathrm{~cm}^{3}$. E m cada horizonte, foram col etadas duas amostras, perfazendo um total de 35 amostras, uma vez que do Cambissolo Háplico (Quadro 1) só foi col etado o horizonte A. Esse material foi, então, saturado por água e, em seguida, submetido a um $\psi$ de-0,01 MPa em mesa de tensão, conforme Klute (1986). Posteriormente, determinou-se a densidade do solo (Ds), de acordo com Blake \& Hartge (1986), após secagem do material em estufa a $105^{\circ} \mathrm{C}$, por 24 horas.

Os procedimentos estatísticos utilizados foram: (a) PROC MEANS (SAS, 1991), para caracterizar os dados; (b) PROC CORR (SAS, 1991), para estabel ecer a correlação entre as variáveis, e (c) PROC REG (SAS, 1991), para desenvolver regressões múltiplas entre propriedades físicas e características rotinei ramente determinadas em levantamentos de

\section{Quadro 1. Classificação e localização dos perfis modais dos solos utilizados no estudo}

\begin{tabular}{|c|c|c|}
\hline Classificação & Localização & Material de origem \\
\hline Planossolo Hidromórfico & Pelotas (RS) & sedimentos areno-argil losos do Grupo Patos, Quaternário \\
\hline Podzólico Bruno-Acinzentado(1) & Canguçu (RS) & $\begin{array}{l}\text { migmatitos do Sub-grupo Cambaí, Grupo Porongos, Pré- } \\
\text { Cambriano }\end{array}$ \\
\hline Argissolo Amarelo & Pinheiro Machado (RS) & $\begin{array}{l}\text { migmatitos do Sub-grupo Cambaí, Grupo Porongos, Pré- } \\
\text { Cambriano }\end{array}$ \\
\hline Chernossolo Ebânico & Bagé (RS) & folhelhos sílticos do Grupo Passa Dois, Permiano \\
\hline Planossolo Hidromórfico & Bagé (RS) & fol hel hos da Formação I rati, Grupo Passa Dois, Permiano \\
\hline Luvissolo Hipocrômico & Bagé (RS) & $\begin{array}{l}\text { migmatitos do Sub-Grupo Cambaí, Grupo Porongos, Pré- } \\
\text { Cambriano }\end{array}$ \\
\hline Luvissolo Hipocrômico & Bagé (RS) & fol hel hos da Formação I rati, Grupo Passa Dois, Permiano \\
\hline Podzólico Vermel ho-Amarelo Álico(1) & Santa Maria (RS) & $\begin{array}{l}\text { arenitos sílticos das Formações Santa Maria e Rosário do Sul, } \\
\text { Sub-Grupo Rio do Rastro, Triássico }\end{array}$ \\
\hline Luvissolo Hipocrômico & São Sepé (RS) & $\begin{array}{l}\text { siltitos areno-argilosos da Formação Palermo, Grupo } \\
\text { Tubarão, Permiano }\end{array}$ \\
\hline Latossolo Vermelho & Tupanciretã (RS) & arenitos da Formação Tupanciretã, Terciário \\
\hline Latossolo Vermelho & Ibirubá (RS) & rochas basálticas do Grupo São Bento, J uro-Cretáceo \\
\hline Latossolo Bruno & Muitos Capões (RS) & rochas basálticas do Grupo São Bento, J uro-Cretáceo \\
\hline Latossolo Bruno & Vacaria (RS) & rochas basálticas do Grupo São Bento, J uro-Cretáceo \\
\hline Cambissolo Háplico & Lages (SC) & $\begin{array}{l}\text { Rochas vul cânicas ácidas (riodacito) do Grupo São Bento, } \\
\text { J uro-Cretáceo }\end{array}$ \\
\hline Nitossolo Háplico & Lages (SC) & rochas basálticas, Grupo São Bento, J uro-Cretáceo \\
\hline Terra Bruna Estruturada Álica(1) & Lebon Régis (SC) & rochas basálticas do Grupo São Bento, J uro-Cretáceo \\
\hline Latossolo Vermelho Ácrico & Canoinhas (SC) & $\begin{array}{l}\text { fol hel hos síltico-argilosos da Formação Rio Bonito, Grupo } \\
\text { Guatá, Super Grupo Tubarão, Permiano }\end{array}$ \\
\hline Podzólico Vermelho-Escuro(1) & Otacílio Costa (SC) & $\begin{array}{l}\text { siltitos da Formação Rio do Rastro, Grupo Passa Dois, } \\
\text { Permiano }\end{array}$ \\
\hline
\end{tabular}

(1) Classificação de acordo com EMBRAPA (1988), pois não foi possível a classificação de acordo com EMBRAPA (1999). 
Quadro 2. Resultados analíticos dos atributos dos solos analisados

\begin{tabular}{|c|c|c|c|c|c|c|c|c|c|c|c|c|c|}
\hline \multirow[b]{2}{*}{ Classificação do solo } & \multirow[b]{2}{*}{ Hor. } & \multicolumn{12}{|c|}{ Característica do solo } \\
\hline & & MO & Dp & Ds & $\theta c c$ & ӨрMP & $\theta_{\text {AD }}$ & AG & AF & $\mathbf{S}$ & $\mathbf{A}$ & $\mathrm{Al}_{2} \mathrm{O}_{3}$ & $\mathrm{Fe}_{2} \mathrm{O}_{3}$ \\
\hline & & $\%$ & \multicolumn{2}{|c|}{$-\mathrm{g} \mathrm{cm}^{-3}-$} & \multicolumn{3}{|c|}{$\mathrm{cm}^{3} \mathrm{~cm}^{-3}$} & \multicolumn{6}{|c|}{$\mathrm{g} \mathrm{kg}^{-1}$} \\
\hline Planossolo Hidromórfico & $\begin{array}{l}\text { Ap } \\
\text { Btgf }\end{array}$ & $\begin{array}{l}1,97 \\
0,67\end{array}$ & $\begin{array}{l}2,56 \\
2,60\end{array}$ & $\begin{array}{l}1,51 \\
1,38\end{array}$ & $\begin{array}{l}0,22 \\
0,34\end{array}$ & $\begin{array}{l}0,11 \\
0,28\end{array}$ & $\begin{array}{l}0,12 \\
0,06\end{array}$ & $\begin{array}{l}285 \\
193\end{array}$ & $\begin{array}{l}356 \\
214\end{array}$ & $\begin{array}{l}258 \\
215\end{array}$ & $\begin{array}{l}101 \\
378\end{array}$ & $\begin{array}{r}19 \\
109\end{array}$ & $\begin{array}{r}7 \\
38\end{array}$ \\
\hline Podzólico Bruno-Acinzentado(1) & $\begin{array}{l}A p \\
B\end{array}$ & $\begin{array}{l}2,84 \\
1,21\end{array}$ & $\begin{array}{l}2,53 \\
2,63\end{array}$ & $\begin{array}{l}1,35 \\
1,50\end{array}$ & $\begin{array}{l}0,32 \\
0,32\end{array}$ & $\begin{array}{l}0,17 \\
0,26\end{array}$ & $\begin{array}{l}0,15 \\
0,06\end{array}$ & $\begin{array}{l}266 \\
224\end{array}$ & $\begin{array}{l}203 \\
158\end{array}$ & $\begin{array}{l}285 \\
202\end{array}$ & $\begin{array}{l}246 \\
416\end{array}$ & $\begin{array}{r}71 \\
134\end{array}$ & $\begin{array}{l}26 \\
44\end{array}$ \\
\hline Argissolo Amarelo & $\begin{array}{l}\text { Ap } \\
\text { Bt1 }\end{array}$ & $\begin{array}{l}2,33 \\
1,19\end{array}$ & $\begin{array}{l}2,60 \\
2,63\end{array}$ & $\begin{array}{l}1,35 \\
1,48\end{array}$ & $\begin{array}{l}0,20 \\
0,31\end{array}$ & $\begin{array}{l}0,13 \\
0,29\end{array}$ & $\begin{array}{l}0,07 \\
0,03\end{array}$ & $\begin{array}{l}442 \\
276\end{array}$ & $\begin{array}{l}215 \\
100\end{array}$ & $\begin{array}{l}221 \\
188\end{array}$ & $\begin{array}{l}122 \\
436\end{array}$ & $\begin{array}{r}38 \\
146\end{array}$ & $\begin{array}{l}21 \\
38\end{array}$ \\
\hline Chernossolo Ebânico & $\begin{array}{l}A p \\
B\end{array}$ & $\begin{array}{l}3,93 \\
1,95\end{array}$ & $\begin{array}{l}2,60 \\
2,53\end{array}$ & $\begin{array}{l}1,48 \\
1,54\end{array}$ & $\begin{array}{l}0,37 \\
0,23\end{array}$ & $\begin{array}{l}0,22 \\
0,21\end{array}$ & $\begin{array}{l}0,15 \\
0,02\end{array}$ & $\begin{array}{l}21 \\
12\end{array}$ & $\begin{array}{l}475 \\
369\end{array}$ & $\begin{array}{l}256 \\
264\end{array}$ & $\begin{array}{l}248 \\
355\end{array}$ & $\begin{array}{l}46 \\
82\end{array}$ & $\begin{array}{l}25 \\
32\end{array}$ \\
\hline Planossolo Hidromórfico & $\begin{array}{l}\text { Ap } \\
\text { Btg1 }\end{array}$ & $\begin{array}{l}1,78 \\
1,05\end{array}$ & $\begin{array}{l}2,53 \\
2,60\end{array}$ & $\begin{array}{l}1,32 \\
1,64\end{array}$ & $\begin{array}{l}0,28 \\
0,37\end{array}$ & $\begin{array}{l}0,09 \\
0,33\end{array}$ & $\begin{array}{l}0,19 \\
0,04\end{array}$ & $\begin{array}{l}274 \\
206\end{array}$ & $\begin{array}{l}312 \\
227\end{array}$ & $\begin{array}{l}313 \\
210\end{array}$ & $\begin{array}{l}101 \\
357\end{array}$ & $\begin{array}{l}17 \\
86\end{array}$ & $\begin{array}{r}8 \\
29\end{array}$ \\
\hline Luvissolo Hipocrômico & $\begin{array}{l}\mathrm{Ap} \\
\mathrm{Bt}\end{array}$ & $\begin{array}{l}2,59 \\
1,97\end{array}$ & $\begin{array}{l}2,50 \\
2,56\end{array}$ & $\begin{array}{l}1,37 \\
1,04\end{array}$ & $\begin{array}{l}0,27 \\
0,38\end{array}$ & $\begin{array}{l}0,15 \\
0,28\end{array}$ & $\begin{array}{l}0,13 \\
0,11\end{array}$ & $\begin{array}{l}382 \\
185\end{array}$ & $\begin{array}{r}212 \\
77\end{array}$ & $\begin{array}{l}243 \\
227\end{array}$ & $\begin{array}{l}163 \\
511\end{array}$ & $\begin{array}{r}44 \\
170\end{array}$ & $\begin{array}{l}23 \\
59\end{array}$ \\
\hline Luvissolo Hipocrômico & $\begin{array}{l}\mathrm{Ap} \\
\mathrm{Bt}\end{array}$ & $\begin{array}{l}2,52 \\
1,62\end{array}$ & $\begin{array}{l}2,60 \\
2,53\end{array}$ & $\begin{array}{l}1,53 \\
1,39\end{array}$ & $\begin{array}{l}0,35 \\
0,43\end{array}$ & $\begin{array}{l}0,20 \\
0,34\end{array}$ & $\begin{array}{l}0,14 \\
0,09\end{array}$ & $\begin{array}{l}221 \\
168\end{array}$ & $\begin{array}{l}197 \\
145\end{array}$ & $\begin{array}{l}418 \\
393\end{array}$ & $\begin{array}{l}164 \\
294\end{array}$ & $\begin{array}{l}32 \\
70\end{array}$ & $\begin{array}{l}15 \\
25\end{array}$ \\
\hline Podzólico Vermel ho-Amarelo Álico(1) & $\begin{array}{l}\text { Ap } \\
\text { Bt1 }\end{array}$ & $\begin{array}{l}0,95 \\
0,50\end{array}$ & $\begin{array}{l}2,63 \\
2,67\end{array}$ & $\begin{array}{l}1,66 \\
1,76\end{array}$ & $\begin{array}{l}0,19 \\
0,24\end{array}$ & $\begin{array}{l}0,07 \\
0,13\end{array}$ & $\begin{array}{l}0,11 \\
0,11\end{array}$ & $\begin{array}{l}537 \\
401\end{array}$ & $\begin{array}{l}245 \\
186\end{array}$ & $\begin{array}{l}158 \\
168\end{array}$ & $\begin{array}{r}60 \\
245\end{array}$ & $\begin{array}{l}13 \\
74\end{array}$ & $\begin{array}{r}8 \\
26\end{array}$ \\
\hline Luvissolo Hipocrômico & $\begin{array}{l}\mathrm{Ap} \\
\mathrm{Bt}\end{array}$ & $\begin{array}{l}1,95 \\
1,48\end{array}$ & $\begin{array}{l}2,53 \\
2,63\end{array}$ & $\begin{array}{l}1,27 \\
1,33\end{array}$ & $\begin{array}{l}0,28 \\
0,41\end{array}$ & $\begin{array}{l}0,13 \\
0,31\end{array}$ & $\begin{array}{l}0,15 \\
0,11\end{array}$ & $\begin{array}{l}136 \\
103\end{array}$ & $\begin{array}{l}370 \\
286\end{array}$ & $\begin{array}{l}331 \\
238\end{array}$ & $\begin{array}{l}163 \\
373\end{array}$ & $\begin{array}{l}30 \\
89\end{array}$ & $\begin{array}{l}16 \\
29\end{array}$ \\
\hline Latossolo Vermelho & $\begin{array}{l}\text { Ap } \\
\text { Bw }\end{array}$ & $\begin{array}{l}1,29 \\
0,52\end{array}$ & $\begin{array}{l}2,78 \\
2,67\end{array}$ & $\begin{array}{l}1,36 \\
1,51\end{array}$ & $\begin{array}{l}0,22 \\
0,29\end{array}$ & $\begin{array}{l}0,10 \\
0,18\end{array}$ & $\begin{array}{l}0,11 \\
0,11\end{array}$ & $\begin{array}{l}200 \\
148\end{array}$ & $\begin{array}{l}499 \\
466\end{array}$ & $\begin{array}{r}99 \\
123\end{array}$ & $\begin{array}{l}202 \\
263\end{array}$ & $\begin{array}{l}58 \\
86\end{array}$ & $\begin{array}{l}24 \\
34\end{array}$ \\
\hline Latossolo Vermel ho & $\begin{array}{l}\text { A1 } \\
\text { Bw1 }\end{array}$ & $\begin{array}{l}3,28 \\
1,19\end{array}$ & $\begin{array}{l}2,74 \\
2,78\end{array}$ & $\begin{array}{l}1,04 \\
1,15\end{array}$ & $\begin{array}{l}0,36 \\
0,44\end{array}$ & $\begin{array}{l}0,25 \\
0,34\end{array}$ & $\begin{array}{l}0,11 \\
0,10\end{array}$ & $\begin{array}{l}78 \\
31\end{array}$ & $\begin{array}{r}137 \\
51\end{array}$ & $\begin{array}{l}212 \\
135\end{array}$ & $\begin{array}{l}573 \\
783\end{array}$ & $\begin{array}{l}173 \\
220\end{array}$ & $\begin{array}{l}187 \\
169\end{array}$ \\
\hline Latossolo Bruno & $\begin{array}{l}\text { Ap } \\
\text { Bw1 }\end{array}$ & $\begin{array}{l}5,34 \\
0,72\end{array}$ & $\begin{array}{l}2,63 \\
2,78\end{array}$ & $\begin{array}{l}1,06 \\
0,91\end{array}$ & $\begin{array}{l}0,42 \\
0,47\end{array}$ & $\begin{array}{l}0,32 \\
0,32\end{array}$ & $\begin{array}{l}0,10 \\
0,15\end{array}$ & $\begin{array}{l}29 \\
16\end{array}$ & $\begin{array}{l}43 \\
27\end{array}$ & $\begin{array}{l}264 \\
151\end{array}$ & $\begin{array}{l}664 \\
806\end{array}$ & $\begin{array}{l}197 \\
219\end{array}$ & $\begin{array}{l}179 \\
197\end{array}$ \\
\hline Latossolo Bruno & $\begin{array}{l}\text { A1 } \\
\text { Bw1 }\end{array}$ & $\begin{array}{l}4,14 \\
1,84\end{array}$ & $\begin{array}{l}2,63 \\
2,82\end{array}$ & $\begin{array}{l}1,12 \\
0,90\end{array}$ & $\begin{array}{l}0,51 \\
0,42\end{array}$ & $\begin{array}{l}0,33 \\
0,30\end{array}$ & $\begin{array}{l}0,18 \\
0,12\end{array}$ & $\begin{array}{l}25 \\
21\end{array}$ & $\begin{array}{l}35 \\
14\end{array}$ & $\begin{array}{l}274 \\
155\end{array}$ & $\begin{array}{l}666 \\
810\end{array}$ & $\begin{array}{l}198 \\
220\end{array}$ & $\begin{array}{l}176 \\
189\end{array}$ \\
\hline Cambissolo Háplico & $A$ & 6,78 & 2,74 & 0,93 & 0,44 & 0,31 & 0,08 & 81 & 58 & 341 & 520 & 177 & 98 \\
\hline Nitossolo Háplico & $\begin{array}{l}\text { A1 } \\
\text { Bt1 }\end{array}$ & $\begin{array}{l}4,59 \\
1,07\end{array}$ & $\begin{array}{l}2,74 \\
2,74\end{array}$ & $\begin{array}{l}1,19 \\
1,38\end{array}$ & $\begin{array}{l}0,45 \\
0,50\end{array}$ & $\begin{array}{l}0,29 \\
0,36\end{array}$ & $\begin{array}{l}0,16 \\
0,14\end{array}$ & $\begin{array}{r}116 \\
89\end{array}$ & $\begin{array}{r}109 \\
99\end{array}$ & $\begin{array}{l}280 \\
151\end{array}$ & $\begin{array}{l}495 \\
661\end{array}$ & $\begin{array}{l}166 \\
191\end{array}$ & $\begin{array}{l}140 \\
155\end{array}$ \\
\hline Terra Bruna Estruturada Álica(1) & $\begin{array}{l}\text { Ap } \\
\text { Bt1 }\end{array}$ & $\begin{array}{l}3,93 \\
1,66\end{array}$ & $\begin{array}{l}2,70 \\
2,82\end{array}$ & $\begin{array}{l}1,18 \\
1,30\end{array}$ & $\begin{array}{l}0,50 \\
0,48\end{array}$ & $\begin{array}{l}0,34 \\
0,37\end{array}$ & $\begin{array}{l}0,16 \\
0,11\end{array}$ & $\begin{array}{l}51 \\
70\end{array}$ & $\begin{array}{l}70 \\
74\end{array}$ & $\begin{array}{l}220 \\
216\end{array}$ & $\begin{array}{l}659 \\
640\end{array}$ & $\begin{array}{l}170 \\
172\end{array}$ & $\begin{array}{l}169 \\
179\end{array}$ \\
\hline Latossolo Vermelho Ácrico & $\begin{array}{l}\text { Ap } \\
\text { Bw1 }\end{array}$ & $\begin{array}{l}3,88 \\
1,67\end{array}$ & $\begin{array}{l}2,56 \\
2,63\end{array}$ & $\begin{array}{l}1,00 \\
0,85\end{array}$ & $\begin{array}{l}0,44 \\
0,37\end{array}$ & $\begin{array}{l}0,21 \\
0,19\end{array}$ & $\begin{array}{l}0,23 \\
0,18\end{array}$ & $\begin{array}{r}107 \\
66\end{array}$ & $\begin{array}{l}209 \\
207\end{array}$ & $\begin{array}{l}146 \\
127\end{array}$ & $\begin{array}{l}538 \\
600\end{array}$ & $\begin{array}{l}198 \\
212\end{array}$ & $\begin{array}{l}82 \\
96\end{array}$ \\
\hline Podzólico Vermel ho-Escuro(1) & $\begin{array}{l}\mathrm{Ap} \\
\mathrm{Bt} 2\end{array}$ & $\begin{array}{l}4,93 \\
0,93\end{array}$ & $\begin{array}{l}2,67 \\
2,63\end{array}$ & $\begin{array}{l}0,92 \\
1,18\end{array}$ & $\begin{array}{l}0,40 \\
0,48\end{array}$ & $\begin{array}{l}0,26 \\
0,33\end{array}$ & $\begin{array}{l}0,15 \\
0,15\end{array}$ & $\begin{array}{l}42 \\
23\end{array}$ & $\begin{array}{r}121 \\
94\end{array}$ & $\begin{array}{l}231 \\
196\end{array}$ & $\begin{array}{l}606 \\
687\end{array}$ & $\begin{array}{l}184 \\
232\end{array}$ & $\begin{array}{l}76 \\
92\end{array}$ \\
\hline
\end{tabular}

(1) MO = teor de matéria orgânica; $\mathrm{Dp}=$ Densidade de partículas; Ds = Densidade do solo; $\theta_{\mathrm{CC}}=$ conteúdo de água a -0,01 MPa de potencial de água no solo; $\mathrm{q}_{\mathrm{PMP}}=$ conteúdo de água a $-1,5 \mathrm{MPa}$ de potencial de água no solo; $\theta_{\mathrm{AD}}=\theta_{\mathrm{CC}}-\theta_{\mathrm{PMP}} ; \mathrm{AG}=$ areia grossa; $\mathrm{AF}=$ areia fina; $\mathrm{S}=$ silte; $\mathrm{A}=$ argila; $\mathrm{Al}_{2} \mathrm{O}_{3}=$ teor de óxidos de alumínio; $\mathrm{Fe}_{2} \mathrm{O}_{3}=$ teor de óxidos de ferro.

solos. O procedimento STEPWISE (SAS, 1991) selecionou as variáveis que entraram nas equações de regressão com $5 \%$ de significância. A variável independente "horizonte" foi introduzida no modelo como variável qualitativa e codificada com o valor 0 (zero), para o horizonte (A), e o val or 1 (um), para o horizonte B. Deacordo com N eter et al. (1989), este procedimento permite fazer uma estimativa mais precisa dos coeficientes, visto que a totalidade dos dados éanalisada em conjunto e, dessa forma, maior número de graus de liberdade é associado com o quadrado médio do erro do modelo. No caso da densidade do solo, por exemplo, os dados foram ajustados ao seguinte model $\mathrm{o:}$

$$
\mathrm{Ds}=\mathrm{a}+\mathrm{b}\left(\mathrm{Al}_{2} \mathrm{O}_{3}\right)
$$

$\mathrm{Na}$ equação 1 , a foi cal culado como demonstrado na equação 2 : 


$$
\mathrm{a}=\left(\mathrm{a} 0+\mathrm{a} 1^{*} \text { horizonte }\right)
$$

Desta forma, nos casos em que houve influência significativa do horizontena propriedadeanalisada, foram geradas duas equações: uma em que $a=a 0$ e outra em quea $=\mathrm{a} 0+\mathrm{a} 1$. No caso em que não houve influência da variável "horizonte", somente foi gerada uma equação. O mesmo procedimento foi utilizado para as outras propriedades físicas analisadas.

\section{RESULTADOS E DISCUSSÃO}

A estatística descritiva das variáveis consideradas nos model os é apresentada no quadro 3.

A maior parte dos atributos dos solos apresenta grande amplitude de variação, fundamental para o estabel ecimento de relações. Esta variação podeser explicada pela gênese diferenciada dos perfis estudados, decorrente de materiais de origem distintos, além de climas e relevos específicos (Quadro 1).

No intuito de demonstrar as relações univariadas existentes entre as propriedades dos solos, foi determi nada, primei ramente, a correlação entre elas (Quadro 4).

A densidade do solo foi negativamente correlacionada com $\mathrm{Al}_{2} \mathrm{O}_{3}$, argila, $\mathrm{Fe}_{2} \mathrm{O}_{3}$ e matéria orgânica e positivamente correlacionada com areia grossa e fina. Relações similares entre Ds ematéria orgânica eDs etextura do sol o foram demonstradas nos trabalhos desenvolvidos por Alexander (1980), Adams (1983), Rawls (1983), Silva et al. (1997) e Bernoux et al. (1998). Em solos tropicais, teores elevados de óxidos de ferro e alumínio estão associados à formação de uma estrutura granular

Quadro 3. Número de observações (n), valores médios (X), desvios-padrão (s), coeficientes de variação (CV), valores míni mos (Mín.) e máxi mos (Máx.) dos atributos dos solos consideradas no estudo

\begin{tabular}{|c|c|c|c|c|c|c|}
\hline Atributos do solo(1) & $\mathbf{n}$ & $\mathbf{x}$ & $\mathbf{s}$ & C.V. (\%) & Mínimo & Máximo \\
\hline Ds, $\mathrm{g} \mathrm{cm}^{-3}$ & 35 & 1,28 & 0,24 & 18,75 & 0,85 & 1,76 \\
\hline $\mathrm{Dp}, \mathrm{g} \mathrm{cm}^{-3}$ & 35 & 2,64 & 0,09 & 3,41 & 2,50 & 2,82 \\
\hline$\theta_{\mathrm{cc}}, \mathrm{cm}^{3} \mathrm{~cm}^{-3}$ & 35 & 0,36 & 0,097 & 26,94 & 0,19 & 0,51 \\
\hline$\theta_{\mathrm{PMP}}, \mathrm{cm}^{3} \mathrm{~cm}^{-3}$ & 35 & 0,24 & 0,089 & 37,08 & 0,07 & 0,37 \\
\hline$\theta_{\mathrm{AD}}, \mathrm{cm}^{3} \mathrm{~cm}^{-3}$ & 35 & 0,12 & 0,047 & 39,17 & 0,02 & 0,23 \\
\hline Areia Grossa, \% & 35 & 15,78 & 13,46 & 85,30 & 1,20 & 53,70 \\
\hline Areia Fina, \% & 35 & 19,04 & 13,28 & 69,75 & 1,40 & 49,90 \\
\hline Silte, \% & 35 & 22,60 & 7,47 & 33,05 & 9,90 & 41,80 \\
\hline Argila, \% & 35 & 42,59 & 22,53 & 52,90 & 6,00 & 81,00 \\
\hline MO, \% & 35 & 2,29 & 1,53 & 66,81 & 0,50 & 6,78 \\
\hline $\mathrm{Al}_{2} \mathrm{O}_{3}, \mathrm{~g} \mathrm{~kg}^{-1}$ & 35 & 124,77 & 71,71 & 57,47 & 13,00 & 232,00 \\
\hline $\mathrm{Fe}_{2} \mathrm{O}_{3}, \mathrm{~g} \mathrm{~kg}^{-1}$ & 35 & 77,63 & 66,76 & 86,00 & 7,00 & 197,00 \\
\hline
\end{tabular}

(1) Ds = Densidade do solo; $\mathrm{Dp}=$ Densidade de partículas; $\theta_{\mathrm{CC}}=$ conteúdo de água a -0,01 MPa de potencial de água no solo; $\theta_{\mathrm{PMP}}=$ conteúdo de água a -1,5 MPa de potencial de água no solo; $\theta_{\mathrm{AD}}=\theta_{\mathrm{CC}}-\theta_{\mathrm{PMP}} ; \mathrm{MO}=$ teor de matéria orgânica; $\mathrm{Al}_{2} \mathrm{O}_{3}=$ teor de óxidos de alumínio; $\mathrm{Fe}_{2} \mathrm{O}_{3}=$ teor de óxidos de ferro.

Quadro 4. Coeficientes de correlação linear entre propriedades físicas e características de solos rotineiramente determinadas em levantamentos pedológicos

\begin{tabular}{|c|c|c|c|c|c|c|c|}
\hline \multirow{2}{*}{ Propriedade física(1) } & \multicolumn{7}{|c|}{ Característica do solo } \\
\hline & Argila & Silte & AG & AF & MO & $\mathrm{Al}_{2} \mathrm{O}_{3}$ & $\mathrm{Fe}_{2} \mathrm{O}_{3}$ \\
\hline Ds, $\mathrm{g} \mathrm{cm}^{-3}$ & $-0,71 * *$ & NS & $0,62^{* *}$ & $0,52 * *$ & $-0,48 * *$ & $-0,74^{* *}$ & $-0,66 * *$ \\
\hline $\mathrm{Dp}, \mathrm{g} \mathrm{cm}^{-3}$ & $0,62 * *$ & $-0,47^{* *}$ & $-0,39 *$ & $-0,39 *$ & NS & $0,57^{* *}$ & $0,71^{* *}$ \\
\hline$\theta_{\mathrm{cc}}, \mathrm{cm}^{3} \mathrm{~cm}^{-3}$ & $0,81^{* *}$ & NS & $-0,73^{* *}$ & $-0,68 * *$ & $0,39 *$ & $0,79 * *$ & $0,74^{* *}$ \\
\hline$\theta_{\mathrm{PMP}}, \mathrm{cm}^{3} \mathrm{~cm}^{-3}$ & $0,80 * *$ & NS & $-0,67 * *$ & $-0,69 * *$ & NS & $0,74^{* *}$ & $0,67^{* *}$ \\
\hline$\theta_{\mathrm{AD}}, \mathrm{cm}^{3} \mathrm{~cm}^{-3}$ & NS & NS & NS & NS & $0,48 * *$ & NS & NS \\
\hline
\end{tabular}

${ }^{(1)}$ Ds = Densidade do solo; $\mathrm{Dp}=$ Densidade de partículas; $\theta_{\mathrm{CC}}=$ conteúdo de água a -0,01 MPa de potencial de água no solo; $\theta_{\mathrm{PMP}}=$ conteúdo de água a -1,5 MPa de potencial de água no solo; $\theta_{\mathrm{AD}}=\theta_{\mathrm{CC}}-\theta_{\mathrm{PMP}} ; \mathrm{AG}=$ conteúdo de areia grossa; $\mathrm{AF}=$ conteúdo de areia fina; $\mathrm{MO}=$ teor de matéria orgânica; $\mathrm{Al}_{2} \mathrm{O}_{3}=$ teor de óxidos de alumínio; $\mathrm{Fe}_{2} \mathrm{O}_{3}=$ teor de óxidos de ferro; * = significativo a $5 \%$; ** = significativo a $1 \%$; NS = não-significativo. 
típica, que determina, normalmente, menores valores de Ds (Schwertmann \& Taylor, 1977; Ferreira et al., 1999a,b).

Os óxidos de ferro e alumínio são importantes para a agregação do solo. Funcionam como agentes deligação entreas partículas minerais, condicionando o tipo de estrutura do solo (Pinheiro-Dick \& Schwertmann, 1996; I gwe et al., 1999; Muggler et al., 1999). Apesar disso, segundo al guns autores, os óxidos de ferro nem sempreinfluenciam a agregação (Desphande et al., 1968; Muggler et al., 1997; Muggler et al., 1999) e, em al guns casos, o efeito dos sesquióxidos pode ser atribuído à interação entre a matéria orgânica e os óxidos e, ou, o teor de argila (Guérif, 1990; Bartoli et al., 1992). Chen et al. (1998) analisaram dados de solos de diversas partes do mundo (incluindo sol os do Brasil) econcluíram quea textura, especificamente o teor de argila, foi o maior determinante das variações de Ds.

A densidade das partículas correlacionou-se positivamente com argila, $\mathrm{Al}_{2} \mathrm{O}_{3}$ e $\mathrm{Fe}_{2} \mathrm{O}_{3}$ e negativamente com silte e areia grossa e fina. A influência dos óxidos de ferro na Dp é esperada para sol os subtropicais etropicais, os quais contêm esses minerais dentro da fração argila e apresentam densidade específica elevada (Schwertmann \& Taylor, 1977).

No caso da capacidade de campo e do ponto de murcha permanente, ocoeficiente de correl ação mais el evado foi obtido com a fração argila. No entanto, valores elevados também foram observados com $\mathrm{Al}_{2} \mathrm{O}_{3}, \mathrm{Fe}_{2} \mathrm{O}_{3}$, areia fina eareia grossa. Para os óxidos, a correlação foi positiva e, para a areia, negativa. Estreita dependência da umidade na $\theta_{\mathrm{Cc}}$ e $\theta_{\mathrm{PMP}}$ em relação à textura também foi observada para solos do estado deSão Paul o por Arruda et al . (1987). Silva \& Kay (1997) verificaram quea água, em diferentes potenciais mátricos, estava diretamenterelacionada com o teor de argila, Ds e teor de carbono orgânico dos solos. Nesteestudo, não foi constatada influência da matéria orgânica no $\theta_{\mathrm{PMP}}$ e, para o $\theta_{\mathrm{CC}}$, a correlação foi significativa a $5 \%$.

A água disponível $\left(\theta_{\mathrm{AD}}\right)$ apresentou coeficiente de correlação significativo (48 \%) apenas com matéria orgânica. Este comportamento pode ser atribuído à el evada capacidade de retenção de água da matéria orgânica, conformemencionado por Silva \& Kay (1997).

Os resultados da análise de regressão múltipla entre as variáveis dependentes (Ds, Dp, $\theta_{\mathrm{CC}}, \theta_{\mathrm{PMP}} \mathrm{e}$ $\theta_{A D}$ ) eas variáveis independentes (Horizonte, Silte, Argila, $\mathrm{MO}, \mathrm{Al}_{2} \mathrm{O}_{3}$ e $\mathrm{Fe}_{2} \mathrm{O}_{3}$ ) encontram-se nos quadros 5 e 6 . O quadro 5 mostra a contribuição parcial ( $R^{2}$ parcial) de cada uma das variáveis independentes (características), e o quadro 6, as equações de regressão para cada propriedade do solo analisada.

Para a Ds, a equação gerada inclui $\mathrm{Al}_{2} \mathrm{O}_{3} \mathrm{e}$ horizonte (Quadro 6). Os óxidos dealumínio, quando na forma de gibbsita e associados a outros óxidos, podem influenciar a agregação do solo, sendo um dos fatores condicionantes do tipo e tamanho de estrutura (Ferreira et al., 1999a). Os óxidos de alumínio geralmente estão associados à maior estabilidade da estrutura granular, com agregados mais individualizados, o que resulta numa menor Ds (Schwertmann \& Taylor, 1977; Ferreira et al., 1999a,b). A menor Ds do horizonte A pode estar associada às modificações da estrutura impostas pelo sistema radicular da vegetação, além da influência da maior atividade biológica. Os model os de Chen et al. (1998) e Silva et al. (1997) revelam apenas a influência da textura e matéria orgânica na Ds, embora esses autores não mencionem se avaliaram a influência dos óxidos nos solos testados.

O teor de óxidos de ferro foi a característica que mais influenciou a Dp. A equação indica que

Quadro 5. Coeficientes de determinação parciais e níveis de significância das variáveis independentes nas equações de regressão obtidas

\begin{tabular}{|c|c|c|c|c|c|c|c|}
\hline \multirow{2}{*}{ Propriedade física estimada(1) } & \multicolumn{6}{|c|}{ Variável independente } & \multirow{2}{*}{$\mathbf{R}^{2}$ tota } \\
\hline & Horizonte & Silte & Argila & MO & $\mathrm{Al}_{2} \mathrm{O}_{3}$ & $\mathrm{Fe}_{2} \mathrm{O}_{3}$ & \\
\hline Ds, $\mathrm{g} \mathrm{cm}^{-3}$ & $0,14 * *$ & NS & NS & NS & $0,55^{* *}$ & NS & 0,69 \\
\hline $\mathrm{Dp}, \mathrm{g} \mathrm{cm}^{-3}$ & NS & $0,09 *$ & NS & NS & NS & $0,50 * *$ & 0,59 \\
\hline$\theta \mathrm{cc}, \mathrm{cm}^{3} \mathrm{~cm}^{-3}$ & NS & $0,13 * *$ & $0,66 * *$ & NS & NS & NS & 0,79 \\
\hline$\theta_{P M P}, \mathrm{~cm}^{3} \mathrm{~cm}^{-3}$ & $0,08 * *$ & $0,09 * *$ & $0,64^{* *}$ & NS & NS & NS & 0,81 \\
\hline$\theta_{A D}, \mathrm{~cm}^{3} \mathrm{~cm}^{-3}$ & $0,24 * *$ & NS & NS & NS & $0,18^{* *}$ & NS & 0,42 \\
\hline
\end{tabular}

\footnotetext{
${ }^{(1)}$ Ds = Densidade do solo; $\mathrm{Dp}=$ Densidade de partículas; $\theta_{\mathrm{CC}}=$ conteúdo de água a $-0,01 \mathrm{MPa}$ de potencial de água no solo; $\theta_{\mathrm{PMP}}=$ conteúdo de água a -1,5 MPa de potencial de água no sol o; $\theta_{A D}=\theta_{C C}-\theta_{P M P} ; \mathrm{MO}=$ teor de matéria orgânica; $\mathrm{Al}_{2} \mathrm{O}_{3}=$ teor de óxidos de alumínio; $\mathrm{Fe}_{2} \mathrm{O}_{3}=$ teor de óxidos de ferro; * =significativo a $5 \%$; ** =significativo a $1 \%$; $\mathrm{NS}$ = variáveis que não foram significativas nos modelos.
} 


\section{Quadro 6. Equações de regressão obti das para as propriedades físicas do solo analisadas e coeficiente de determinação do modelo gerado}

\begin{tabular}{|c|c|c|c|c|}
\hline Propriedade física estimada(1) & Horizonte & Equações de regressão & $\mathbf{R}^{2}$ total & $\mathbf{P}>\mathbf{F}$ \\
\hline Ds, $\mathrm{g} \mathrm{cm}^{-3}$ & $\begin{array}{l}\text { A } \\
B\end{array}$ & $\begin{array}{l}\text { Ds }=1,56-0,003 \mathrm{Al}_{2} \mathrm{O}_{3} \\
\text { Ds }=1,75-0,003 \mathrm{Al}_{2} \mathrm{O}_{3}\end{array}$ & $\begin{array}{l}0,69 \\
0,69\end{array}$ & $\begin{array}{l}<0,0001 \\
<0,0001\end{array}$ \\
\hline $\mathrm{Dp}, \mathrm{g} \mathrm{cm}^{-3}$ & $A$ e $B$ & $\mathrm{Dp}=2,66-0,004$ Silte $+0,00085 \mathrm{Fe}_{2} \mathrm{O}_{3}$ & 0,59 & $<0,0001$ \\
\hline$\theta_{\mathrm{PMP}}, \mathrm{cm}^{3} \mathrm{~cm}^{-3}$ & $\begin{array}{l}\text { A } \\
B\end{array}$ & $\begin{array}{l}\theta_{\mathrm{PMP}}=-0,031+0,005 \text { Silte }+0,003 \text { Argila } \\
\theta_{\mathrm{PMP}}=0,024+0,005 \text { Silte }+0,003 \text { Argila }\end{array}$ & $\begin{array}{l}0,81 \\
0,81\end{array}$ & $\begin{array}{l}<0,0001 \\
<0,0001\end{array}$ \\
\hline$\theta_{\mathrm{CC}}, \mathrm{cm}^{3} \mathrm{~cm}^{-3}$ & $A$ e $B$ & $\theta_{\mathrm{CC},}=0,081+0,005$ Silte $+0,004$ Argila & 0,79 & $<0,0001$ \\
\hline$\theta_{\mathrm{AD}}, \mathrm{cm}^{3} \mathrm{~cm}^{-3}$ & $\begin{array}{l}\text { A } \\
B\end{array}$ & $\begin{array}{l}\theta_{A D}=0,115+0,0003 \mathrm{Al}_{2} \mathrm{O}_{3} \\
\theta_{A D}=0,055+0,0003 \mathrm{Al}_{2} \mathrm{O}_{3}\end{array}$ & $\begin{array}{l}0,42 \\
0,42\end{array}$ & $\begin{array}{l}0,0030 \\
0,0030\end{array}$ \\
\hline
\end{tabular}

aumentos no teor desilteresultaram em diminuição dos valores da Dp (Quadro 6), embora tenha sido baixa a correlação entre esses dois atributos (Quadro 5). A maior contribuição parcial foi dada pelo $\mathrm{Fe}_{2} \mathrm{O}_{3}$. A relação direta entre óxidos de ferro e Dp depende das maiores densidades específicas apresentadas por esses minerais e pela elevada propor ção em que se encontram na maior parte dos sol os estudados (Schwertmann \& Taylor, 1977).

As equações obtidas para as variáveis $\theta_{\text {PMP }}$ e $\theta_{C C}$ apresentaram elevados coeficientes de determinação, sendo $64 \%$ da variação da $\theta_{\text {PMP }}$ e 66 \% da variação da $\theta_{\mathrm{Cc}}$ explicadas pela variação do teor de argila. A contribuição da fração silte foi pequena (Quadro 5). A variável qualitativa "horizonte" foi significativa apenas para o modelo da $\theta_{\mathrm{PMP}}$. A diferença de $\theta_{\mathrm{PMP}}$ entre os horizontes $A$ e B pode estar relacionada com a influência de íons e sais na retenção de água do solo (por exemplo, $\mathrm{Na}^{+}$nos Planossolos Hidromórficos).

De maneira geral, esses resultados concordam com os obtidos por Piedgeon (1972), para solos de Uganda; Silva \& Kay (1997), para sol os do Canadá, eVan den Berg et al. (1997), para Ferralsols/Oxisols e solos relacionados da América do Sul, África e sudeste da Ásia. Arruda et al . (1987). Van den Berg et al. (1997) geraram equações para retenção deágua para solos das regiões Sul e Sudeste do Brasil, mas utilizaram como variável independente a soma argila + silte. No presente estudo, a contribuição parcial das variáveis silte e argila foi altamente significativa, optando-se por incluí-las separadamente no modelo.

O coeficiente de determinação total para a equação obtida para $\theta_{A D}$ foi baixo, embora tenha sido altamente significativo (Quadro 6). Valores baixos de coeficientes de determinação para $\theta_{A D}$ já tinham tinham sido relatados por Van den Berg et al. (1997), para solos tropicais. A ausência de relação de $\theta_{A D}$ com al gumas das frações granulométricas tem sido atribuída ao fato de que os parâmetros $\theta_{C C}$ e $\theta_{P M P}, a$ partir dos quais é calculada a $\theta_{A D}$, são obtidos em laboratório e, muitas vezes, com amostras deformadas, ocasionando alteração da estrutura, fator este determinante da quantidade de água retida pel o solo e disponível para as plantas. Com a perda da estrutura natural do perfil dificilmentesão simuladas as condições de movimentação de água que ocorrem no campo (Arruda et al., 1987). A água disponível $\left(\theta_{A D}\right)$ foi positivamenteinfluenciada pelos óxidos de alumínio e pela variável qualitativa "horizonte". A diferença de $\theta_{\mathrm{AD}}$ entre os horizontes pode estar relacionada com variações no tipo e tamanho de estrutura dos solos que acarretam diferente distribuição e tamanho de poros (Barral et al., 1998).

Demaneira geral, observou-se que os coeficientes de determinação dos modelos obtidos foram altamente significativos (Quadro 6), apesar de requererem um número pequeno de variáveis. Isso demonstra que, a princípio, o comportamento das propriedades físicas estudadas está relacionado com poucas características dos solos, rotineiramente determinadas em levantamentos pedológicos.

\section{CONCLUSÕES}

1. A densidade do solo foi influenciada pelos óxidos de alumínio e tipo de horizonte considerado; a densidade de partículas, por silteeóxidos deferro.

2. A umidade na capacidade de campo foi influenciada por silte e argila; o ponto de murcha 
permanente, por silte, argila e tipo de horizonte, e o teor de água disponível, por óxidos de alumínio.

3. Tais resultados podem auxiliar o desenvolvimento de funções de pedotransferência para estimar propriedades físicas dos sol os da regiãoSul doBrasil.

\section{LITE RATURA CITADA}

ADAMS, W.A. The effect of organic matter on the bulk and true densities of some uncultivated podzolic soils. J. Soil Sci., 24:10-17, 1983.

ALEXANDER, E.B. Bulk densities of California soils in relation to other soil properties. Soil Sci. Soc. A. J ., 44:689-692, 1980.

ARRUDA, F.B.; J ÚLIO J r., J . \& OLIVEIRA, J .B. Parâmetros de solo para cálculo de água disponível com base na textura do solo. R. Bras. Ci. Solo, 11:11-15, 1987.

BARRAL, M.T.; ARIAS, M. \& GUÉRIF, J. Effects of iron and organic matter on the porosity and structural stability of soil aggregates. Soil Till. Res., 46:261-272, 1998.

BARTOLI, F.; BURTIN, G. \& GUÉRIF, J. Influence of organic matter on aggregation in Oxisols rich in gibbsite or in goethite. II. Clay dispersion, aggregate strength and water stability. Geoderma, 54:259-274, 1992.

BERNOUX, M.; ARROUAYS, D.; CERRI, C. VOLKOFF, B. \& J OLIVET, C. Bulk densities of Brazilian Amazon soil related to other soil properties. Soil Sci. Soc. Am. J., 62:743-749, 1998.

BLAKE, G.R. \& HARTGE, K.H. Bulk density. In: KLUTE, A., ed. Methods of soil analysis. 2.ed. Madison, American Society of Agronomy, 1986. v.1, p.363-375.

BOUMA, J. Using soil survey data for quantitative land evaluation. Adv. Soil Sci., 9:177-213, 1989.

CHEN, Y.; TESSIER, S. \& ROUFFIGNAT, J. Soil bulk density estimation for tillage systems and soil textures. Trans. Am. Soc. Agric. Eng., 41:1601-1610, 1998.

DESPHANDE, T.L.; GREENLAND, D.J . \& QUIRK, J .P. Charges in soil properties associated with the removal of iron and aluminum oxides. J. Soil Sci., 19:108-122, 1968.

EMPRESA BRASILEIRA DE PESQUISA AGROPECUÁRIA EMBRAPA. Centro Nacional de Pesquisa de Solos. Sistema Brasileiro de Classificação de solos. Rio de J aneiro, Ministério da Agricultura, 1999. 257p.

EMPRESA BRASILEIRA DE PESQUISA AGROPECUÁRIA EMBRAPA. Serviço Nacional de Levantamento e Conservação de Solos. Manual de métodos de análise de solo. Rio de J aneiro, Ministério da Agricultura, 1979. Não paginado.

EMPRESA BRASILEIRA DE PESQUISA AGROPECUÁRIA EMBRAPA. Serviço Nacional de Levantamento e Conservação de Solos. Critérios para distinção de classes de solos e de fases de unidades de mapeamento: normas em uso pelo SNLC. Rio de J aneiro, Ministério da Agricultura, 1988. 67p. (Documentos SNLCS, 11)
FERREIRA, M.M.; FERNANDES, B. \& CURI, N. Influência da fração argila nas propriedades físicas de $L$ atossol os da região sudeste do Brasil. R. Bras. Ci. Solo, 23:515-524, 1999b.

FERREIRA, M.M.; FERNANDES, B. \& CURI, N. Mineralogia da fração argila e estrutura de $L$ atossol os da região sudeste do Brasil. R. Bras. Ci. Solo, 23:507-514, 1999a.

GUÉRIF, J. Factors influencing compaction-induced increases in soil strength. Soil Till. Res., 16:167-178, 1990.

IGWE, C.A.; AKAMIGBO, F.O.R. \& MBAGWU, J S.C. Chemical and mineralogical properties of soils in southeastern Nigeria in relation to aggregate stability. Geoderma, 92:111123, 1999.

INSTITUTO AGRONÔMICO DE CAMPINAS - IAC. Métodos de análise química, mineralógica e física de solos do Instituto Agronômico de Campinas. Campinas, 1986. 94p. (Bol etim Técnico, 106)

KLUTE, A. Water retention: laboratory methods. In: KLUTE, A., ed. Methods of soil analisys. 2 ed. Madison, American Society of Agronomy, 1986. v.1, p.635-660.

MUGGLER, C.C.; PAPE, TH. \& BUURMAN, P. Laser grain-size determination in soil genetic studies 2 . Clay content, clay formation, and aggregation in some Brazilian Oxisols. Soil Sci., 162:219-228, 1997.

MUGGLER, C.C.; VAN GRIETHUYSEN, C.; BUURMAN, P. \& PAPE, T. Aggregation, organic matter, and iron oxide morphology in Oxisols from Minas Gerais, Brazil. Soil Sci., 164:759-770, 1999.

NETER, J.; WASSERMAN, W. \& KUTNER, M. H. Applied linear regression models, 2 ed. Boston, 1989. 667p.

PACHEPSKI, Ya.A. \& RAWLS, W.J . Accuracy and reliability of pedotransfer functions as affected by grouping soils. Soil Sci. Soc. Am. J ., 63:1748-1757, 1999.

PIDGEON, J.D. The measurement and prediction of available water capacity of Ferrallitic soils in Uganda. J. Soil Sci., 23:431-441, 1972.

PINHEIRO-DICK, D. \& SCHWERTMANN, U. Microaggreggates from Oxisols and Inceptisols: dispersion through selective dissolutions and physicochemical treatments. Geoderma, 74:49-63, 1996.

REUNIÃO DE CORRELAÇÃO, CLASSIFICAÇÃO E APLICAÇÃO DE LEVANTAMENTOS DE SOLOS, 6. 2000, Colombo. Guia de excursão de estudos de sol os nos estados do Rio Grande do Sul, Santa Catarina e Paraná. Colombo: Embrapa Floresta/Rio deJ aneiro: Embrapa Solos/Campinas, IAC, 2000. 222p. (Embrapa Florestas, Documentos, 222p)

RAWLS, W.J . Estimation soil bulk density from particle size analysis and organic matter content. Soil Sci., 135:123-125, 1983 (Note).

SAS INSTITUTE. Statiscal Analysis System Institute - SAS/ STAT Procedure guide for personal computers. Version 5, SAS I nst. Cary, NC. 1991.

SCHWERTMANN, U. \& TAYLOR, R.M. I ron Oxides. In: DIXON, J.B., ed. Minerals in soil environments. Madison, Soil Science Society of America, 1977. 948p.

SI LVA, A.P. \& KAY, B.D. Estimating the least limiting water range of soils from properties and management. Soil Sci. Soc. Am. J., 61:877-883, 1997. 
SILVA, A.P.; KAY, B.D. \& PERFECT, E. Management versus inherent properties effects on bulk density and relative compaction. Soil Till. Res., 44:81-93, 1997.

TIETJE, O. \& TAPKENHINRICHS, M. Evaluation of pedotransfer functions. Soil Sci. Soc. Am. J ., 57:1088-1095, 1992.

TOMASELLA, J .; HODNETT, M.G. \& ROSSATO, L. Pedotransfer functions for the estimation of soil water retention in brazilian soils. Soil Sci. Soc. Am. J ., 64:327-338, 2000.

Van den BERG, M.; KLAMT, E.; van REEUWIJ K, L.P. \& SOMBROEK, W.G. Pedotransfer functions for the estimation of moisture retention characteristics of Ferralsols and related soils. Geoderma, 78:161-180, 1997.
Van GENUCHTEN, M.T. A closed-form equation for predicting the hydraulic conductivity of unsaturated soils. Soil Sci. Soc. Am. J ., 44:892-898, 1980.

VETTORI, L. Métodos de análises de solos. Rio de J aneiro, Ministério da Agricultura, 1969. 24p. (Boletim Técnico, 7)

WAGENET, R.J .; BOUMA, J \& GROSSMAN, R.B. Minimun data sets for use of soil survey information in soil interpretive models. In: MAUSBACH, M.J . \& WILDING, L.P., eds. Spatial variabilities of soils and landforms. Madison, Soil Science Society of America, 1991. (Special Publication, 28) 
N.F.B. GIAROLA et al. 